\title{
Asymmetric Temperature Load Method for Simulation Study of Wing Leading Edge in Flight Deicing with MFC
}

\author{
Wei Wang and Zhichun Yang \\ Department of Aerospace Structure Engineering, School of Aeronautics, \\ Northwestern Polythechnical University, Xi'an 710072, China \\ wwang@nwpu.edu.cn
}

\begin{abstract}
Leading edge icing jeopardizes the aerodynamic performance of wing and the fly safety of airplane. Macro fiber composite(MFC) piezoelectric actuator possess favorable flexibility and large force output ability, which makes MFC quite suitable for the excitation of large curvature thin-walled structures such as wing leading edge. In this study, MFC is adopted for leading edge in flight deicing application. Key challenges of leading edge in flight deicing with MFC were investigated by finite element methodology and simulation approach, which include fundamental analysis of vibration deicing method, leading edge deicing excitation mode and MFC placement scheme determination. Base on thermal-elastic analogy modeling theory, asymmetric temperature load modeling method is proposed for the special actuation effect simulation of twist type MFC for deicing. Simulation results demonstrate the third natural vibration mode can be utilized for leading edge deicing with lowest energy consumption and twist type MFC has great potential for leading edge in flight deicing application.
\end{abstract}

Keywords: leading edge deicing, natural mode vibration, MFC piezoelectric actuator, asymmetric temperature load method

\section{Introduction}

Excellent aerodynamic performance of aircraft wing comes from its well designed airfoil shape. However leading edge icing during flight will change this designed shape evidently, debase the aerodynamic performance of aircraft wing. In some worst case it may cause air crash, because ice will greatly decrease the wing stall speed and make the stall speed more easily to be exceeded even the pilot could notice. Airplane icing problem research can be traced back to the end of 20s to early 30s of 20th century, the first icing wind tunnel came up during the World War II. In 1978, Glenn (later Lewis) research center was founded in NASA to research the influence of icing on lift to drag ratio of airfoil and even the performance of the whole airplane. Nowadays in flight deicing has become a major concern for civil and military aircraft. Accumulated ice should be removed before it affects aircraft wing performance and flight safety. Currently electrical heating and electrical impulse method are two kinds of practical methods for in flight deicing [1]. However, both of these two methods have their drawbacks. Efficiency of electrical elements used in the electrical heating method will bring much constrain on deicing speed, and high energy consumption is another problem. Thermal deicing methods require around $10 \mathrm{KW}$ of power to deice $1 \mathrm{~m}^{2}$ of the ice surface [2]. 
There are also difficulties in laying out the electrical elements inside aircraft structure. Those drawback bring much constrain to the application of electrical heating method. Electrical impulse method may possess faster deicing speed but the weight cost because of the additional impulse generating devices will reach an adverse level.

Recently, Suresh et al proposed an in flight deicing concept for leading edge using Quick Pack Actuators, and their experiment results has proved this method is energy efficient $[2,3]$. In addition, Ramanathan et al studied helicopter blades deicing using piezoelectric actuator [4], which utilized the same deicing mechanism. Macro fiber composite piezoelectric actuator (MFC) was invented by NASA Langley Research Center. The most important advantage of MFC is its excellent flexibility and that makes it quite suitable for the excitation and vibration control of large curvature thin-walled structure. Considering the leading edge has large curvature and thin-walled structure features, MFC should have great potential for leading edge in flight deicing application. In this study, leading edge in flight deicing method using MFC piezoelectric actuator is presented. Simulation is conducted in time domain to validate the feasibility of MFC excited vibration deicing method.

\section{Fundamental of Leading Edge Deicing by Structure Vibration}

Ice layer can attach to structure surface because the existence of a thin layer called adhesive layer between the underside of ice layer and structure surface [2]. Shear and tension strength of this adhesive layer has large difference in value, mean value of the shear strength is $1.65 \mathrm{MPa}$, mean value of the tension strength is $300 \mathrm{MPa}$. Since the shear strength of this adhesive layer is much smaller, it is much easier to be destroyed. Once the adhesive layer is broken, high speed airflow will blow the ice block away from the wing's leading edge. Now the problem is how to obtain the stress we need to break the adhesive layer. Speaking of generating this shear stress, utilizing structure vibration is an ideally controllable option. And the required shear stress for deicing is only needed to be higher than $1.65 \mathrm{MPa}$, which means with properly control this local vibration brings extremely small influence on the fatigue life of leading edge structure.

Fundamental of in flight vibration deicing method for leading edge structure can be described as making the structure vibrating with certain mode frequency and shape, generating enough shear stress level on structure surface to break the adhesive layer between structure surface and ice layer. Then high-speed airflow will strike and blow the ice block away. Leading edge deicing can be achieved.

\subsection{Leading edge structure modeling for deicing analysis}

In this section, finite element model (FEM) of leading edge structure was established to illustrate the mechanism of vibration deicing method. NACA0012 airfoil was chosen to construct the leading edge structure. Coordinates of selected point from NACA0012 airfoil for the modeling of leading edge structure are listed in Table 1, by linking those points the profile of leading edge is formed. Geometry configuration for leading edge FEM is obtained by gliding this profile along axial $\mathrm{Z}$ for $300 \mathrm{~mm}$. Material of this thin-walled leading edge structure is defined as LY12, elastic modular is $70000 M P a$, Poisson's ratio is 0.3 , density is $2700 \mathrm{Kg} / \mathrm{m}^{3}$, and thickness is $1 \mathrm{~mm}$. FEM of leading edge configuration is show in Figure 1.

Using this FEM, shear stress of element 7 for the first five natural modes of leading edge structure were extracted and listed in the left side of Table 2. Location of element 7 can be seen in Figure 1. From the data listed in Table 2, it can be found that the shear stress for the third natural mode of leading edge structure of element 7 exceed $1.65 \mathrm{MPa}$, which will 
satisfy the deicing shear stress requirement. According to this result, within the first five natural modes the third natural mode and the fifth natural mode can both be chosen for vibration deicing application.

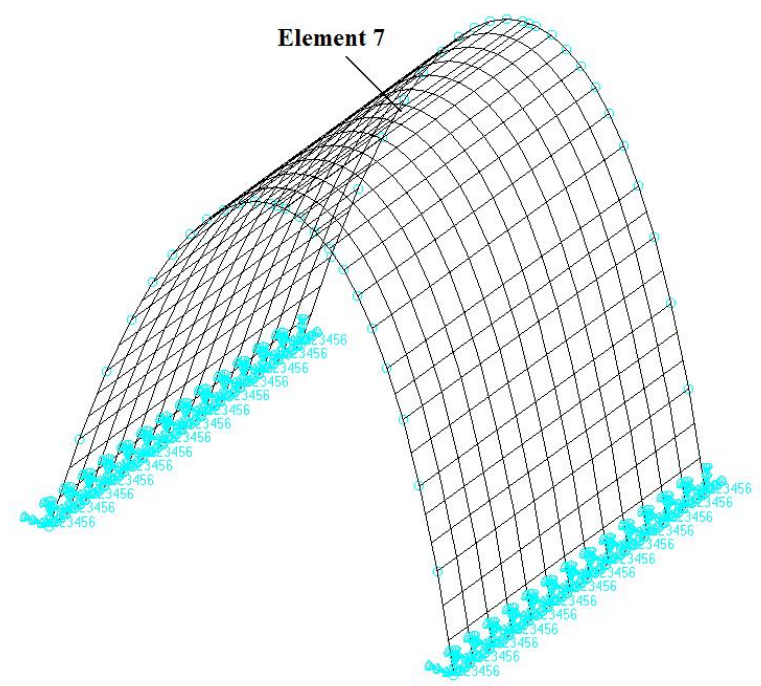

Figure 1. Finite element model of leading edge structure

Table 1. Coordinates of selected point from NACA 0012 airfoil for the modeling of leading edge structure

\begin{tabular}{|c|c|c|c|c|c|c|c|c|}
\hline No. & $\mathrm{X}(\mathrm{mm})$ & $\mathrm{Y}(\mathrm{mm})$ & No. & $\mathrm{X}(\mathrm{mm})$ & $\mathrm{Y}(\mathrm{mm})$ & No. & $\mathrm{X}(\mathrm{mm})$ & $\mathrm{Y}(\mathrm{mm})$ \\
\hline 1 & 317.04 & 199.25 & 9 & 43.04 & 85.25 & 17 & 54.54 & -7.25 \\
\hline 2 & 246.54 & 181.75 & 10 & 34.54 & 73.75 & 18 & 69.54 & -19.25 \\
\hline 3 & 190.04 & 164.25 & 11 & 28.54 & 61.75 & 19 & 89.04 & -31.75 \\
\hline 4 & 146.54 & 148.75 & 12 & 26.04 & 50.25 & 20 & 114.04 & -44.75 \\
\hline 5 & 114.04 & 134.25 & 13 & 26.04 & 39.25 & 21 & 146.54 & -58.75 \\
\hline 6 & 89.04 & 121.25 & 14 & 28.54 & 27.75 & 22 & 190.04 & -74.75 \\
\hline 7 & 69.54 & 108.75 & 15 & 34.54 & 15.75 & 23 & 246.54 & -91.75 \\
\hline 8 & 54.54 & 97.25 & 16 & 43.04 & 4.25 & 24 & 317.04 & -109.75 \\
\hline \multicolumn{8}{|c|}{$\mathrm{Z}=0 \mathrm{~mm}$} \\
\hline
\end{tabular}

\subsection{Leading edge structure modeling with ice}

In the previous analysis ice layer has not been considered in the FEM. In this section FEM for wing leading edge structure with ice covering its local surface was implemented. Laminated composite plate modeling approach was adopted for the ice covering region modeling. Using this approach the mass and stiffness contribution of ice layer to the leading edge structure would be taken into account at the same time. Density of ice is defined as $800 \mathrm{Kg} / \mathrm{m}^{3}$, averaged modulus of elasticity is defined as $53 \mathrm{MPa}$. Ice layer thickness is defined as $5 \mathrm{~mm}$. 


\section{Table 2. Shear stress of element 7 for different natural vibration modes of leading edge structure}

\begin{tabular}{|c|c|c||c|c|c|}
\hline \multicolumn{2}{|c||}{ Result from leading edge FEM analysis } & \multicolumn{3}{c|}{ Result from leading edge with ice layer } \\
\hline $\begin{array}{c}\text { Mode } \\
\text { Number }\end{array}$ & $\begin{array}{c}\text { Frequency } \\
(\mathrm{Hz})\end{array}$ & $\begin{array}{c}\text { Shear tress on the } \\
\text { surface of } \\
\text { element 7 (MPa) }\end{array}$ & $\begin{array}{c}\text { Mode } \\
\text { Number }\end{array}$ & $\begin{array}{c}\text { Shear stress } \\
\text { Frequency } \\
\text { (Hz) }\end{array}$ & $\begin{array}{c}\text { between ice layer } \\
\text { surface and } \\
\text { leading edge skin } \\
\text { surface of } \\
\text { element 7 (MPa) }\end{array}$ \\
\hline 1 & 21.1 & $2.89842 \mathrm{E}-07$ & 1 & 21.0 & $2.21135 \mathrm{E}-06$ \\
\hline 2 & 51.8 & $-6.19422 \mathrm{E}-07$ & 2 & 52.0 & $7.10403 \mathrm{E}-05$ \\
\hline $\mathbf{3}$ & $\mathbf{6 1 . 4}$ & $\mathbf{1 . 2 2 9 1 5 E + 0 1}$ & $\mathbf{3}$ & $\mathbf{6 1 . 4}$ & $\mathbf{5 . 0 5 3 3 1 E + 0 0}$ \\
\hline 4 & 92.6 & $-8.49318 \mathrm{E}-06$ & 4 & 92.6 & $-1.03546 \mathrm{E}-05$ \\
\hline 5 & 106.2 & $2.70505 \mathrm{E}+02$ & 5 & 106.5 & $-2.21916 \mathrm{E}+02$ \\
\hline
\end{tabular}

Extracted shear stress value of element 7 for the first five natural modes is listed in the right side of Table 2. It can be seen that the shear stress on the aluminum skin surface of the iced wing leading edge is about 5.1MPa, when leading edge vibrates by its third natural mode the required shear stress level for deicing will be achieved.

When ice layer is taken count into the leading edge structure FEM, it can be concluded that the third natural mode and the fifth mode are both can be utilized as the deicing mode for leading edge deicing. This conclusion is only made for the first five modes investigated here. The next step is to determine which one is more suitable for leading edge in flight deicing application.

\section{Energy Consumption Analysis and Key Parameter Optimization}

According to the aforementioned fundamental analysis of the vibration deicing method for wing leading edge structure, excitation mode for deicing application can be selected as the candidate modes which also can be called deicing mode. Piezoelectric actuator MFC is adopted to excite wing leading edge structure to vibrate with the deicing mode.

The piezoelectric actuator energy consumption can be described as [3]

$$
P=\frac{1}{2} n \omega V^{2} C_{i}
$$

in which, $C_{i}=$ the effective capacity of the MFC piezoelectric actuator, $n=$ the number of MFC piezoelectric actuators are used, $\omega=$ frequency of driving signal, $V=$ amplitude of driving voltage.

It can be seen from the above Eq. (1), for a certain piezoelectric actuator and given driving voltage amplitude, energy consumption of the piezoelectric actuator is proportional to the driving frequency. From the energy consumption point of view, deicing mode selection should consider the lowest natural mode of leading edge structure which satisfies the deicing 
requirement. According to the previous analysis, the lowest order of natural modes, which can provide the deicing shear stress level(greater than $1.65 \mathrm{MPa}$ ), is the third natural mode of leading edge structure, so the third natural mode of the leading edge structure should be chosen to be the deicing mode.

Tow basic principles for the piezoelectric excitation deicing mode selection can be concluded as follow, (1) the chosen mode should provide a certain shear stress level for deicing requirement; (2) Energy consumption should be as low as possible, which means the lowest natural mode which satisfies rule no. (1) should be chosen.

In addition, in order to energize the chosen deicing mode effectively, attachment location of MFC needs to be optimized according to the shear stress distribution of the deicing mode.

The acknowledged principle for piezoelectric actuator placement indicates MFC should be attached to the region where stress is relatively higher. According to our practical experience, the piezoelectric actuator should also be arranged in the area where the structural stiffness is relatively small, in another word the chosen area should avoid too close to the clamped boundary or local stiffened area of structure.

According to the principle mentioned above and computed shear stress distribution of leading edge structure for the third natural mode, location of MFC is set to the same location as element 7 which can be seen in Figure 2. The corresponding shear stress of this region is relatively higher, and the stiffness is relatively low.

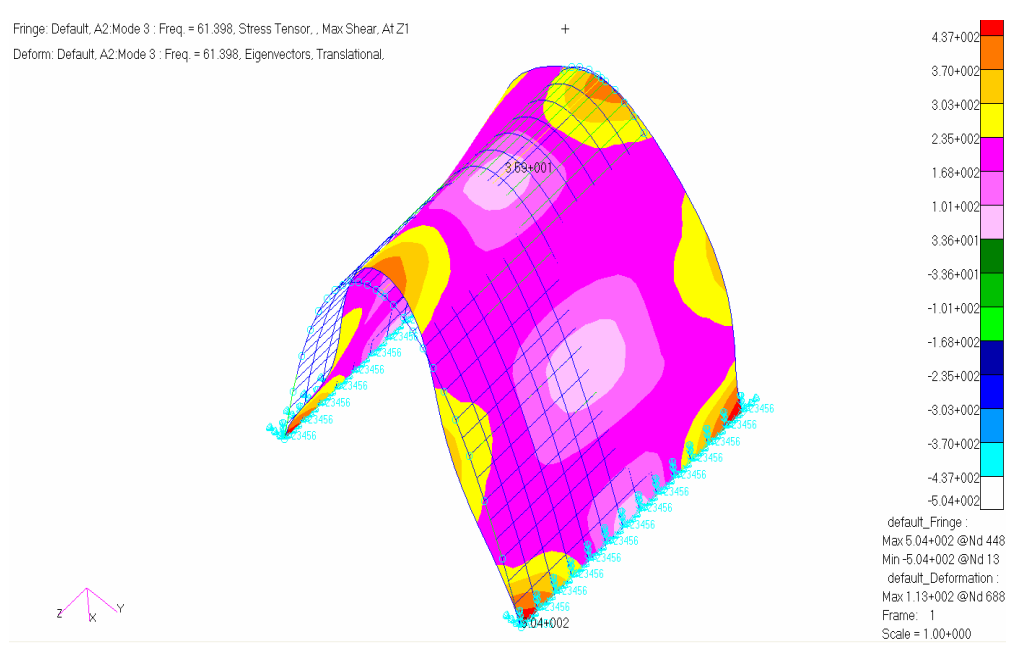

Figure 2. Shear stress distribution for the third natural mode of leading edge structure

\section{MFC Excited Vibration Deicing Method Simulation}

Macro fiber composite (MFC) piezoelectric actuators utilize the inverse piezoelectric effect of piezoelectric materials; the piezoelectric constant $d_{33}$ is utilized. MFC is constructed by piezoelectric fibers with rectangle cross section, which has been polarized in its length direction. In the length direction of the piezoelectric fiber equally spaced electrodes are arranged, these electrodes divided piezoelectric fiber into a number of equal length segments, when driving voltage is imposed to these electrodes, piezoelectric fiber will generate elongation deformation along its length direction. These piezoelectric fibers attached to a substrate which is made of Kapton (polyimide). Deformation of piezoelectric fiber is passed 
to structure surface by this substrate, and strain/stress coupling can be constructed between the MFC and attached structure. Fundamental of MFC structural design is to cut piezoelectric ceramic foil into piezoelectric fiber, makes MFC possessing excellent flexibility compared with traditional PZT piezoelectric patch actuator, which is similar to the design theory of steel wire rope. The excellent flexibility can be seen in Figure 3. Parameters of the piezoelectric material used in MFC are listed in Table 3.

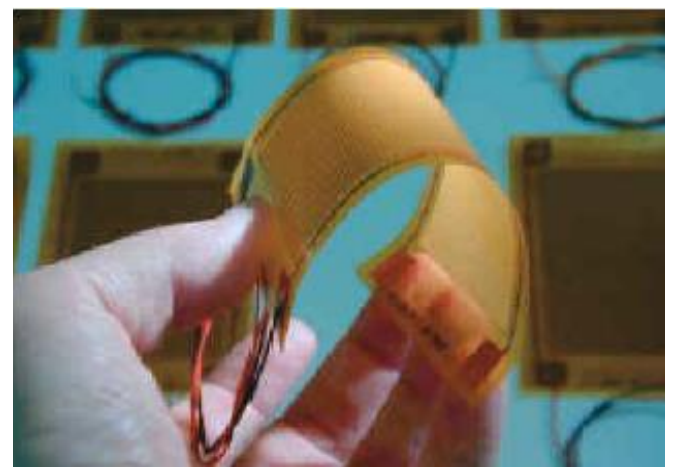

Figure 3. Picture showing the flexibility of MFC piezoelectric actuator [7]

Table 3. Parameters of the piezoelectric material used in MFC

\begin{tabular}{|c|c|c|c|}
\hline Material Parameter & Value & Material Parameter & Value \\
\hline Modulus of Elasticity $E_{1} / \mathrm{Pa}$ & 30.34 & $\begin{array}{c}\text { Modulus of Elasticity } \\
E_{2} / \mathrm{GPa}\end{array}$ & 15.86 \\
\hline $\begin{array}{c}\text { Piezoelectric Constant } \\
d_{33} / 10^{-10} \frac{\mathrm{C}}{\mathrm{N} \cdot m}\end{array}$ & $4.0 \sim 4.6$ & $\begin{array}{c}\text { Piezoelectric Constant } \\
d_{31} / 10^{-10} \frac{\mathrm{C}}{\mathrm{N} \cdot m}\end{array}$ & \\
\hline Poisson's ratio $\gamma_{12}$ & 0.31 & Poisson's ratio $\gamma_{21}$ & 0.16 \\
\hline Density $\mathrm{Kg} / \mathrm{m}^{3}$ & 7800 & electrode spacing $\left(10^{-4} \mathrm{~m}\right)$ & 5.3 \\
\hline
\end{tabular}

Using thermal-elastic analogy method, state space model of structures with integrated piezoelectric actuator can be built. Firstly an artificial coefficient of thermal expansion has to be introduced and defined as $\alpha=d_{31} / t$, where $d_{31}$ represents the piezoelectric strain constant and $t$ is defined as a geometry character value of piezoelectric actuator. For MFC this $t$ should be defined equal to the electrode spacing of the interdigitated electrode of MFC.

The governing equation of vibration system without damping can be written as

$$
\boldsymbol{M} \ddot{x}+\boldsymbol{K} x=f
$$

where $x \in R^{n}$ represents the displacement vector, $f$ is the actuating force, $\boldsymbol{M}$ and $\boldsymbol{K}$ represents the mass and stiffness matrices of the structure in which the mass and stiffness contributions of piezoelectric material has already been considered. Using the theory of thermal-elastic analysis, a Ritz vector $S_{t}$ can be obtained with a unit temperature load applied to the structure. This vector can be equivalent to the Ritz vector induced by a driving voltage applied to the piezoelectric actuator which has been integrated with the structure. In 
general, one Ritz vector is required for each piezoelectric actuator. Then the actuating force can be written as

$$
f=\boldsymbol{K} S_{t} V
$$

where $V$ represents the driving voltage for the actuator.

Thermal-elastic analogy modeling method can be directly used for bend type MFC simulation, general operation is to impose unit symmetric temperature load at the four corner nodes of piezoelectric laminated shell element, as show in the left side of Figure 4. The detailed process of the simulation can be found in our published article [6]. In the other hand, for the simulation of twist type MFC piezoelectric actuator with 45degree piezoelectric fiber arrangement, there haven't been found any proposed method. Based on the thermal-elastic analogy method, asymmetric temperature load method is proposed for the simulation of this particular MFC piezoelectric actuator. Scheme of the proposed approach can be seen in the right side of Figure 4, asymmetric temperature loads were imposed on the corner nodes of the piezoelectric laminated shell element to simulate effect of twist actuation.

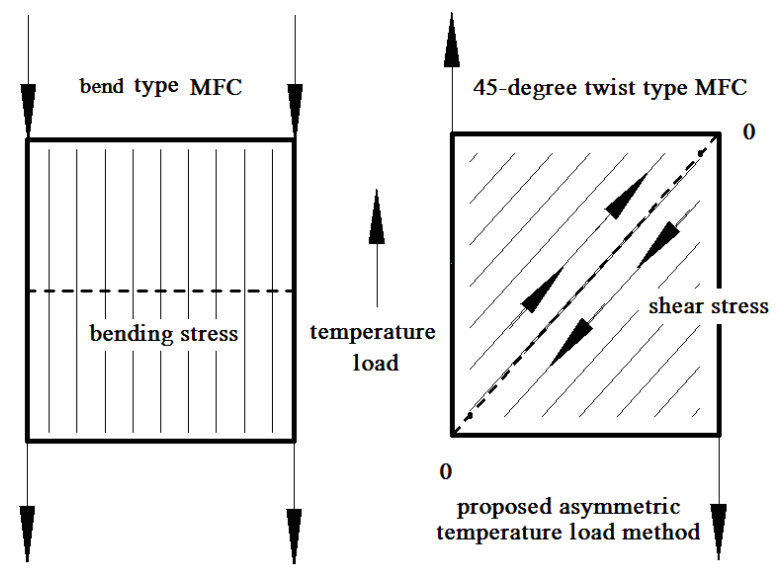

Figure 4. Scheme of modeling method for bend and twist type MFC

At this stage, we could define the first coordinate transformation equation

$$
x=\left[\begin{array}{ll}
\boldsymbol{\Phi} & S_{t}
\end{array}\right]\left\{\begin{array}{c}
z \\
z_{r}
\end{array}\right\}
$$

where $\boldsymbol{\Phi}$ is the normalized eigenvector matrix of Eq. (2) with respect to generalized mass. Substituting Eq. (3) and Eq. (4) into Eq. (2) and multiply both sides of Eq.(2) by $\left[\begin{array}{ll}\boldsymbol{\Phi} & S_{t}\end{array}\right]^{T}$ we can obtain

$$
\left[\begin{array}{cc}
\boldsymbol{I} & \boldsymbol{M}_{r} \\
\boldsymbol{M}_{r}^{T} & \boldsymbol{M}_{r r}
\end{array}\right]\left\{\begin{array}{c}
\ddot{z} \\
\ddot{z}_{r}
\end{array}\right\}+\left[\begin{array}{cc}
\boldsymbol{\Lambda} & \boldsymbol{K}_{r} \\
\boldsymbol{K}_{r}^{T} & \boldsymbol{K}_{r r}
\end{array}\right]\left\{\begin{array}{c}
z \\
z_{r}
\end{array}\right\}=\left\{\begin{array}{c}
\boldsymbol{K}_{r} \\
\boldsymbol{K}_{r r}
\end{array}\right\} V
$$

where $\boldsymbol{M}_{r}=\boldsymbol{\Phi}^{T} \boldsymbol{M} S_{t}, \quad \boldsymbol{M}_{r r}=S_{t}^{T} \boldsymbol{M} S_{t}, \quad \boldsymbol{K}_{r}=\boldsymbol{\Phi}^{T} \boldsymbol{K} S_{t}, \quad \boldsymbol{K}_{r r}=S_{t}^{T} \boldsymbol{K} S_{t}, \boldsymbol{I}$ represents identity matrix. Assume that only the first $m$ order of normal modes are considered, 
then $\boldsymbol{\Phi} \in R^{n \times m}, \boldsymbol{\Lambda} \in R^{m \times m}$. Define the global generalized mass matrix and stiffness matrix as

$$
\overline{\boldsymbol{M}}=\left[\begin{array}{cc}
\boldsymbol{I} & \boldsymbol{M}_{r} \\
\boldsymbol{M}_{r}^{T} & \boldsymbol{M}_{r r}
\end{array}\right], \quad \overline{\boldsymbol{K}}=\left[\begin{array}{cc}
\boldsymbol{\Lambda} & \boldsymbol{K}_{r} \\
\boldsymbol{K}_{r}^{T} & \boldsymbol{K}_{r r}
\end{array}\right]
$$

The Ritz vector obtained from thermal-elastic analysis is a static result which makes Eq. (5) a stiff equation. In order to eliminate the ill condition of the stiff equation, another coordinate transformation has to be made by following definition

$$
\left\{\begin{array}{c}
z \\
z_{r}
\end{array}\right\}=\boldsymbol{W}\left\{\begin{array}{l}
\eta \\
\eta_{r}
\end{array}\right\}
$$

where matrix $\boldsymbol{W}$ is the right singularity matrix of $\overline{\boldsymbol{M}}$, which can be obtained according to Eq. (8)

$$
\overline{\boldsymbol{M}}=\boldsymbol{U}\left[\begin{array}{ll}
\boldsymbol{\sigma} & 0 \\
0 & 0
\end{array}\right] \boldsymbol{W}^{T}
$$

$\boldsymbol{\sigma}$ is the non-zero singularity value of $\overline{\boldsymbol{M}}, \quad \boldsymbol{U}$ is the left singularity matrix of $\overline{\boldsymbol{M}}$.

After the second coordinate transformation was carried out, Eq. (9) is obtained

$$
\left[\begin{array}{cc}
\boldsymbol{\sigma} & 0 \\
0 & 0
\end{array}\right]\left\{\begin{array}{c}
\ddot{\eta}_{\eta_{r}}
\end{array}\right\}+\left[\begin{array}{cc}
\boldsymbol{k} & \boldsymbol{k}_{r} \\
\boldsymbol{k}_{r}^{T} & \boldsymbol{k}_{r r}
\end{array}\right]\left\{\begin{array}{c}
\eta \\
\eta_{r}
\end{array}\right\}=\left\{\begin{array}{c}
b \\
b_{r}
\end{array}\right\} V
$$

where

$$
\left[\begin{array}{cc}
\boldsymbol{k} & \boldsymbol{k}_{r} \\
\boldsymbol{k}_{r}^{T} & \boldsymbol{k}_{r r}
\end{array}\right]=\boldsymbol{U}^{T} \overline{\boldsymbol{K}} \boldsymbol{W},\left\{\begin{array}{c}
b \\
b_{r}
\end{array}\right\}=\boldsymbol{U}^{T}\left\{\begin{array}{l}
\boldsymbol{K}_{r} \\
\boldsymbol{K}_{r r}
\end{array}\right\}
$$

Solve $\eta_{r}$ from Eq. (9)

$$
\eta_{r}=-\boldsymbol{k}_{r r}^{-1} \boldsymbol{k}_{r}^{T} \eta+\boldsymbol{k}_{r r}^{T} b_{r} V
$$

Submit Eq. (11) into the first equation of Eq. (9) and finally we have

$$
\boldsymbol{\sigma} \ddot{\eta}+\left(\boldsymbol{k}-\boldsymbol{k}_{r} \boldsymbol{k}_{r r}^{-1} \boldsymbol{k}_{r}^{T}\right) \eta=\left(b-\boldsymbol{k}_{r} \boldsymbol{k}_{r r}^{-1} \boldsymbol{k}_{r}^{T}\right) V
$$

Eq. (12) is the reduced order model for the vibrating deicing system actuated by MFC. The state space model of the deicing system can be constructed straightforwardly from Eq. (12). With the state space model, deicing simulation work can be carried out conveniently and efficiently.

$$
\begin{aligned}
& \dot{x}(t)=\boldsymbol{A} x(t)+\boldsymbol{B} u(t) \\
& y(t)=\boldsymbol{C} x(t)
\end{aligned}
$$

Eq. (13) is the state equation, where $x(t)$ is the state vector, $u(t)$ is the control (input) vector, $\boldsymbol{A}$ is the state matrix, and $\boldsymbol{B}$ is the input matrix. Eq. (14) is the output equation, where $y(t)$ is the output vector, $\boldsymbol{C}$ is the output matrix. For the leading edge structure with MFC, the matrices of the state space model for this piezoelectric control system can be constructed according to the following equations. 


$$
\begin{gathered}
\boldsymbol{A}=\left[\begin{array}{cc}
0 & \boldsymbol{I} \\
-\boldsymbol{\sigma}^{-1}\left(\boldsymbol{k}-\boldsymbol{k}_{r} \boldsymbol{k}_{r r}^{-1} \boldsymbol{k}_{r}^{T}\right) & -\boldsymbol{\sigma}^{-1} \overline{\boldsymbol{C}}
\end{array}\right] \\
\boldsymbol{B}=\left[\begin{array}{c}
0 \\
\boldsymbol{\sigma}^{-1}\left(b-\boldsymbol{k}_{r} \boldsymbol{k}_{r r}^{-1} \boldsymbol{k}_{r}^{T}\right)
\end{array}\right] \\
\boldsymbol{C}=\left[\begin{array}{ll}
\boldsymbol{\Phi} & S_{t}
\end{array}\right]
\end{gathered}
$$

where $\overline{\boldsymbol{C}}$ is a diagonal matrix, which represents the damping matrix of the system. Deformation vector $S_{t}$ can be obtained by thermal elasticity theory, when the unit temperature load field is applied to certain part of the structure to which the MFC piezoelectric actuator is attached. Flow chart of the thermal-elastic analogy modeling method is illustrated in Figure 5.

Twist type MFC can be used in the twist mode excitation of structure, which can generate a certain level of shear stress on structure surface. In this study, twist type MFC is chosen for vibration deicing application. In twist type MFC, the piezoelectric ceramic thickness is $180 \mu \mathrm{m}$, and piezoelectric macro fiber width is $350 \mu \mathrm{m}$, and the piezoelectric fiber is arranged in 45-degree, scheme of which also can be seen in the right side of Figure 4.

Using the proposed asymmetric temperature load method, state space model for this piezoelectric system can be obtained. Simulation of the application of using the third natural mode vibration to generate the required shear stress in element 7 is implemented.

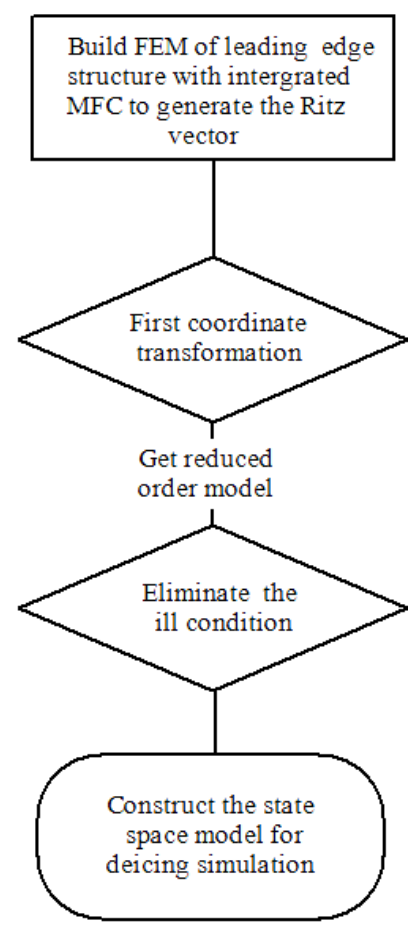

Figure 5. Flow chart of the piezoelectric system modeling for deicing simulation 


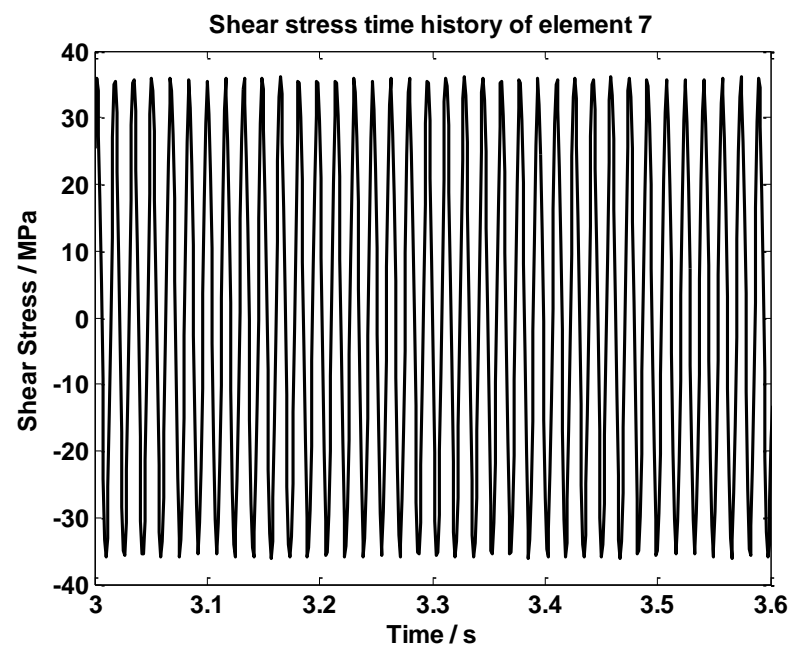

Figure 6. Simulated shear stress time history of element 7 excited by twist type MFC under the third natural mode of leading edge structure with driving voltage amplitude 150 volts

The shear stress amplitude in element 7 of the third natural mode vibration with different amplitude of driving voltage is illustrated in Table 4. Shear stress time history simulation result of element 7, excited by twist type MFC under the third natural mode of leading edge structure with driving voltage amplitude 150 volts is illustrated in Figure 6. Simulation result demonstrates that using twist type MFC, the required shear stress for leading edge deicing can be provided by actuating the chosen deicing mode. It can be found that the amplitude of shear stress is proportional to the driving voltage amplitude.

Table 4. Shear stress amplitude of element 7 for the third natural mode vibration under different amplitude of driving voltages

\begin{tabular}{|c|c|}
\hline $\begin{array}{c}\text { Amplitude of driving } \\
\text { voltage } \\
(\mathrm{V})\end{array}$ & $\begin{array}{c}\text { Shear stress amplitude } \\
\text { of element 7 (MPa) }\end{array}$ \\
\hline 10 & 2.4 \\
\hline 50 & 12 \\
\hline 100 & 24 \\
\hline 150 & 36 \\
\hline 200 & 48 \\
\hline
\end{tabular}

\section{Conclusion}

1) Base on thermal-elastic analogy method asymmetric temperature load method is proposed for twist type MFC modeling. It has been demonstrated in the simulation study of leading edge vibration deicing application.

2) Considering shear stress requirements for deicing and energy consumption of piezoelectric actuator, the third natural mode of leading edge structure can be utilized as the deicing mode for leading edge in flight deicing application. 
3) MFC is very suitable for the excitation of thin-wall structure with large curvature such as wing leading edge, analysis and simulation results demonstrate that twist type MFC has great potential for in flight leading edge vibration deicing application.

\section{Acknowledgements}

This work was supported by the National Natural Science Foundation of China (Grant No. 11072198) and the Foundation of Northwestern Polytechnical University for Fundamental Research (Grant No. JC201102) and the 111 Project of China (Grant No.B07050)

\section{References}

[1] C. A. Martin and J. C. Putt, "Advanced pneumatic impulse ice protection system (PIIP) for aircraft", J. Aircraft, vol. 4, no. 29, (1992), pp. 714-716.

[2] S. V. Venna and Y. J. Lin, "Mechatronic Development of Self-Actuating In-Flight Deicing Structures", IEEE/ASME Transactions on Mechatronics, vol. 11, no. 5, (2006), pp. 585-592.

[3] S. V. Venna, "Development of self-actuated in-flight deicing technology utilizing smart structure concepts", Ph.D. dissertation, University of Akron, (2005).

[4] S. Ramanathan, V. V. Varadhan and V. K. Varadhan, "Deicing of helicopter blades using piezoelectric actuators", J. Smart Structure Material, vol. 3990, no. 281, (2000), pp. 281-292.

[5] B. D. Freed and V. Babusk, "Finite element modeling of composite piezoelectric structures with MSC/NASTRAN", Proceeding of SPIE 4th Annual Symposium on Smart Structures and material: Smart Structure and Integrated Systems, (1997) March San Diego USA, pp. 6767-6788.

[6] W. Wang and Z. C. Yang, "Integrated Simulation and Design Method for Piezoelectric Active Vibration Control System on Fin Model”, J. of System Simulation, vol. 22, no. 800, (2010), pp. 800-803.

[7] H. A. Sodano, "Macro-Fiber Composites for Sensing Actuation and Power Generation", Thesis for Master of Science, Virginia Polytechnic Institute and State Uniersity, (2003).

[8] H. Y. Zhang, C. Q. Bai and Y. P. Shen, "Experimental and Numerical Analysis for Twist Control of Rotor blade model with Macro Fiber Composite (MFC)", Chinese J. of Applied Mechanics, vol. 26, no. 456, (2009), pp. 456-460. 
International Journal of Control and Automation Vol.6, No.6 (2013) 